\title{
RISCO DE QUEDAS EM PACIENTES NO PERÍODO PÓS-OPERATÓRIO
}

Allyne Fortes Vitor', Laísla Alves Moura², Ana Paula Nunes de Lima Fernandes², Fabiane Rocha Botarelli³, Jessica Naiara de Medeiros Araújo ${ }^{4}$, Ingrid Caroline da Costa Vitorino ${ }^{5}$

Enfermeira. Doutora em Enfermagem. Docente da Universidade Federal do Rio Grande do Norte. Natal-RN-Brasil. ${ }^{2}$ Enfermeira. Mestranda em Enfermagem. Universidade Federal do Rio Grande do Norte. Natal-RN-Brasil.

${ }^{3}$ Enfermeira. Mestre em Enfermagem. Docente da Universidade Federal do Rio Grande do Norte. Natal-RN-Brasil. ${ }^{4}$ Discente de enfermagem. Universidade Federal do Rio Grande do Norte. Natal-RN-Brasil.

${ }^{5}$ Enfermeira. Especialista em Enfermagem do Trabalho. Universidade Federal do Rio Grande do Norte. Natal-RN-Brasil.

RESUMO: O estudo objetivou identificar o diagnóstico de enfermagem Risco de quedas em pacientes no período pós-operatório, internados em um hospital universitário e os principais fatores de risco nesta clientela. Tratase de um estudo descritivo e transversal de caráter quantitativo. Dados coletados de outubro a dezembro de 2012, por meio de roteiro estruturado para investigar os dados sociodemográficos e clínicos. Dos 80 pacientes investigados, 86,25\% apresentaram o Diagnóstico de Enfermagem Risco de quedas. Os fatores de risco que mais se destacaram foram: história de quedas, condições pós-operatórias, uso de narcóticos e/ou opiáceos e uso de anti-hipertensivos. Diante da diversidade de fatores de risco, encontrados em pacientes no período pós-operatório, torna-se imperativo a identificação precoce desses por profissionais de saúde, a fim de propor ações preventivas e eficazes que suprimam ou minimizem tais riscos.

DESCRITORES: Enfermagem; Acidentes por quedas; Diagnóstico de enfermagem; Enfermagem perioperatória.

\section{RISK FOR FALLS IN PATIENTS IN THE POSTOPERATIVE PERIOD}

\section{RIESGO DE CAÍDAS EN PACIENTES EN EL PERIODO POSOPERATORIO}

\begin{abstract}
The study aimed to identify the Risk for falls nursing diagnosis and the main risk factors in patients in the postoperative period, at a university hospital. This was a descriptive, cross-sectional study with a quantitative approach. Data were collected from October to December 2012, using a structured script to investigate the sociodemographic and clinical data. Of the 80 patients investigated, $86.25 \%$ presented the Risk for Falls Nursing Diagnosis. The risk factors that stood out were: history of falls, postoperative conditions, use of narcotics and/or opiates and use of antihypertensive medication. Given the diversity of risk factors found in patients in the postoperative period, the early identification of these factors by health professionals is imperative, in order to propose preventive and effective actions that suppress or minimize such risks.
\end{abstract}

DESCRIPTORS: Nursing; Accidental falls; Nursing diagnosis; Perioperative nursing.
RESUMEN: El estudio tuvo la finalidad de identificar el diagnóstico de enfermería Riesgo de caídas en pacientes en el periodo posoperatorio, internados en un hospital universitario y los principales factores de riesgo en esta clientela. Es un estudio descriptivo y transversal de carácter cuantitativo. Datos fueron recogidos de octubre a diciembre de 2012, por medio de guión estructurado para investigar las informaciones sociodemográficas y clínicas. De los 80 pacientes investigados, $86,25 \%$ presentaron Diagnóstico de Enfermería Riesgo de Caídas. Los factores de riesgo que más se destacaron fueron: historia de caídas, condiciones posoperatorias, uso de narcóticos y/o opiáceos y uso de antihipertensivos. Delante de la diversidad de factores de riesgo en pacientes en el periodo posoperatorio, es imperativa la identificación precoz de eses por profesionales de salud, a fin de proponer acciones preventivas y eficaces que supriman o minimizen tales riesgos.

DESCRIPTORES: Enfermería; Accidentes por caídas; Diagnóstico de enfermería; Enfermería perioperatoria. 


\section{INTRODUÇÃO}

A assistência de enfermagem aos pacientes nas instituições hospitalares, nos últimos anos, tem exigido atuação extremamente complexa, a fim de se ofertar um cuidado resolutivo, sobretudo com qualidade. Neste sentido, a segurança dos pacientes ganha relevância e se torna ferramenta imperativa, ao evocar da enfermagem capacidade científica e comprometimento com ações sistêmicas de avaliação e prevenção, na tentativa de viabilizar a redução de desfechos indesejados $^{(1)}$.

No contexto da internação hospitalar considera-se período pós-operatório (PO) desde o momento em que o paciente deixa a sala de cirurgia até o término do acompanhamento pela equipe de saúde, após a sua avaliação final. Por ser uma fase crítica, são necessários cuidados redobrados de toda a equipe de saúde a fim de garantir uma recuperação segura a esta clientela ${ }^{(2)}$.

Os pacientes internados em período pósoperatório estão sujeitos a diversas complicações, dentre elas o risco de quedas. Por sua vez, as quedas estão entre os eventos adversos mais comuns relatados em pacientes hospitalizados. Tal fenômeno torna-se comum nesta população em virtude da própria condição de recuperação da saúde, da mudança de ambiente ou mesmo da terapia medicamentosa adotada ${ }^{(3)}$.

Este evento adverso pode resultar em substancial morbidade para o paciente e, consequente responsabilidade para a instituição( ${ }^{(4)}$. Em virtude deste excesso de morbidade relacionada a quedas no período de internação, a Joint Commission on Accreditation of Healthcare Organizations elenca diversos programas de prevenção de quedas em hospitais como um dos principais objetivos de segurança do paciente. $\mathrm{O}$ primeiro passo para a criação de um programa de prevenção de quedas, de base hospitalar em pacientes cirúrgicos, é entender os fatores de risco que ocasionam este evento adverso no pósoperatório ${ }^{(5)}$.

Assim, para que gastos, injúrias e danos indesejáveis aos pacientes e profissionais sejam evitados, medidas preventivas por parte da enfermagem precisam ser adotadas em curto prazo, na tentativa de reverter esse processo $^{(6-7)}$. Surge, por conseguinte, a necessidade de organização e operacionalização do trabalho da enfermagem quanto ao método, ao pessoal e aos instrumentos fundamentais para efetivar a avaliação dos indivíduos sobre os riscos inerentes à segurança $\mathrm{a}^{(7)}$.

Destarte, torna-se fundamental identificar os fatores de risco que contribuem para o aumento da vulnerabilidade do indivíduo. Remete-se então, à Sistematização da Assistência de Enfermagem (SAE), mediante o Processo de Enfermagem (PE) como ferramenta metodológica para identificar e prevenir tais riscos ${ }^{(8)}$. Nessa perspectiva, o risco de quedas pode ser identificado como um fenômeno da enfermagem reconhecido na linguagem de seus sistemas de classificação por meio do diagnóstico de enfermagem (DE) Risco de Quedas( ${ }^{(9)}$.

Este DE é definido como a suscetibilidade para quedas que podem causar danos físicos. Os fatores de risco que o caracterizam estão dispostos em: ambientais; cognitivos; adultos; crianças; fisiológicos e medicamentos. É pertencente à classe de Lesão Física, componente do Domínio Segurança e Proteção, que por sua vez, corresponde ao indivíduo estar livre de perigo, lesão física ou dano do sistema imunológico, preservação contra perdas e proteção da segurança e seguridade ${ }^{(9)}$.

Diante da relevância do tema para a enfermagem, os pacientes e instituições em identificar e prevenir o evento adverso risco de quedas, surgiu, portanto, a seguinte questão norteadora: como está caracterizado o DE Risco de quedas em pacientes no pós-operatório?

Diante do exposto, o presente estudo objetivou identificar o diagnóstico de enfermagem Risco de quedas em pacientes no período pós-operatório internados em um hospital universitário e os principais fatores de risco nesta clientela.

\section{METODOLOGIA}

Trata-se de um estudo descritivo e transversal, de caráter quantitativo, desenvolvido em um hospital universitário público e de nível terciário localizado município de Natal/ RN, Brasil. A população foi composta por pacientes em pósoperatório, internados nas unidades de clínica cirúrgica do referido hospital.

A amostra foi determinada a partir da 
aplicação de uma fórmula desenvolvida para estudos com populações infinitas e que leva em consideração o nível de confiança, a prevalência e o erro amostral. Foi considerada, portanto, uma população infinita, a prevalência de pacientes atendidos na unidade de clínica cirúrgica desse hospital, um nível de confiança de 95\% e um erro amostral de 5\%.

A fórmula para o cálculo da amostra foi a seguinte: $\mathrm{N}=\left(\mathrm{Za}^{2} \bullet \mathrm{P} \bullet \mathrm{Q}\right) / \mathrm{E} 2$, onde $\mathrm{N}=$ tamanho da amostra; $\mathrm{Za}=$ o nível de confiança; $\mathrm{P}=$ prevalência de pacientes atendidos na unidade de clínica cirúrgica desse hospital; $Q=$ complemento da prevalência (1- P); $\mathrm{E}=\mathrm{o}$ erro amostral. Desta maneira a amostra foi consistida em 80 pacientes.

Para inclusão na amostra foram considerados os seguintes critérios: estar internado no setor de clínica cirúrgica do referido hospital; ter idade igual ou superior a 18 anos; estar em condições físicas e emocionais de responder às perguntas e de ser submetido ao exame físico e estar em condições mínimas de mobilidade. O critério de exclusão da amostra foi: apresentar durante a coleta de dados situações de emergência com risco de morte.

As condições físicas correspondem à capacidade cognitiva do participante, ao qual foi aplicado o Mini Mental State Examination (MMSE) por parte dos examinadores componentes da equipe de pesquisa. $\mathrm{O}$ paciente foi incluído ao obter a seguinte pontuação: $>15$ (se analfabeto); $>22$ (de 1 a 11 anos de estudo) e > 27 (tempo de estudo superior a 11 anos). Com relação às condições emocionais, o próprio paciente participante da pesquisa foi incluído se, e somente se, sentia-se e referia estar emocionalmente apto a fazê-lo no momento da coleta de dados.

Os dados foram coletados de outubro a dezembro de 2012, mediante realização de anamnese, exame físico e consulta ao prontuário. Para a anamnese utilizou-se um roteiro estruturado com base na taxonomia II da NANDA-I, proposto a obter os dados sociodemográficos e clínicos, além de informações relevantes ao risco de quedas. Com posterior consulta ao prontuário, foram extraídas informações como os tipos de medicações utilizadas e exames laboratoriais.

O raciocínio clínico e posterior inferência diagnóstica seguiram o processo de julgamento de Gordon ${ }^{(10)}$ mediante o qual foram realizados, primeiramente, pelos pesquisadores de forma individual para cada paciente e em seguida foi procedido por consenso entre os pesquisadores para indicar a presença do diagnóstico de enfermagem Risco de quedas.

Os fatores de risco do DE Risco de quedas foram investigados conforme sua classificação na NANDA-I, sendo eles: específicos para adultos, fisiológicos, medicamentos. Não foram incluídos no presente estudo os fatores de risco exclusivos para crianças, por não se aplicarem a população de interesse, assim como a categoria de fatores cognitivos, uma vez que foi estabelecido para inclusão no estudo que o indivíduo apresentasse capacidade cognitiva preservada.

Referente aos fatores ambientais percebeu-se uma divergência entre as interpretações por parte dos pesquisadores quanto a essa questão, uns fizeram sua própria observação sobre o ambiente, enquanto outros perguntaram ao entrevistado. Como esse quesito não foi padronizado no questionário e essa limitação só foi percebida na fase de análise dos dados, decidiu-se excluir essa categoria do resultado final.

A análise estatística foi realizada de acordo com os princípios da estatística descritiva e associativa, com obtenção de frequências, médias, desvio-padrão e intervalos de confiança, bem como a aplicação do teste de KolmogorovSmirnov a fim de verificar a normalidade dos achados. Considerou-se um Intervalo de Confiança de 95\% e nível de significância de $p<0,05$. Os dados foram estatisticamente tratados por pacotes estatísticos específicos para os seguintes testes: exato de Fisher, Mann-Whitney, teste-T assumindo variâncias iguais e foi calculado o Coeficiente Phi.

Ressalta-se que a pesquisa foi aprovada pelo Comitê de Ética e Pesquisa da Universidade Federal do Rio Grande do Norte sob o parecer $\mathrm{n}^{\mathrm{o}} 121.028$ e registrado mediante o CAAE 07614812.6.0000.5537, em concordância com as determinações da Resolução no 196/96 do Conselho Nacional de Saúde ${ }^{(11)}$. A cada um dos pacientes foi realizada uma explicação prévia sobre os propósitos do trabalho e a garantia de seu anonimato, bem como sobre a aplicação do roteiro de entrevista e exame físico. Mediante aceitação em participar deste estudo, ocorreu a assinatura do Termo de Consentimento Livre e Esclarecido por parte de cada participante. 


\section{RESULTADOS}

Dentre os participantes, o DE Risco de quedas esteve presente em 69 pacientes $(86,25 \%)$, os dados sociodemográficos relacionados à sua ocorrência estão expostos nas Tabela 1 e 2.

Tabela 1 - Distribuição dos pacientes em pós-operatório, segundo os dados sociodemográficos, clínicos. Natal-RN, 2013

\begin{tabular}{|c|c|c|c|c|c|}
\hline \multirow{3}{*}{ Variáveis } & \multicolumn{4}{|c|}{ Risco de quedas } & \multirow{3}{*}{ Estatísticas } \\
\hline & \multicolumn{2}{|c|}{ Presente } & \multicolumn{2}{|c|}{ Ausente } & \\
\hline & $\mathbf{n}$ & $\%$ & $\mathbf{n}$ & $\%$ & \\
\hline Sexo & & & & & \multirow{4}{*}{$\begin{array}{l}p=0,1011 \\
P h i=0,178 \quad(p= \\
0,112) \\
\text { OR=3,461 }(0,695 \\
-17,221)\end{array}$} \\
\hline Masculino & 39 & 48,75 & 9 & 11,25 & \\
\hline Feminino & 30 & 37,50 & 2 & 2,50 & \\
\hline Total & 69 & 86,25 & 11 & 13,75 & \\
\hline \multicolumn{5}{|l|}{ Estado civil } & \multirow{4}{*}{ 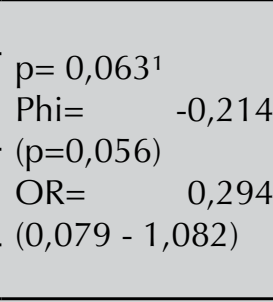 } \\
\hline $\begin{array}{l}\text { Com } \\
\text { companheiro }\end{array}$ & 51 & 63,75 & 5 & 6,25 & \\
\hline $\begin{array}{l}\text { Sem } \\
\text { companheiro }\end{array}$ & 18 & 22,50 & 6 & 7,50 & \\
\hline Total & 69 & 86,25 & 11 & 13,75 & \\
\hline Religião & & & & & \multirow{4}{*}{$\begin{array}{ll}p=0,0051 & \\
P h i= & -0,339 \\
(p=0,002) & \\
\text { OR= } & 0,145 \\
(0,037 & -0,567)\end{array}$} \\
\hline Praticante & 55 & 68,75 & 4 & 5 & \\
\hline Não praticante & 14 & 17,50 & 7 & 8,75 & \\
\hline Total & 69 & 86,25 & 11 & 13,75 & \\
\hline
\end{tabular}

Legenda: ${ }^{1}$ Teste exato de Fisher;

Tabela 2 - Ocorrência do Diagnóstico de Enfermagem Risco de quedas. Natal-RN, 2013

\begin{tabular}{|c|c|c|c|c|c|}
\hline \multirow{3}{*}{ Variáveis } & \multicolumn{4}{|c|}{ Risco de quedas } & \multirow{3}{*}{ Estatísticas } \\
\hline & \multicolumn{2}{|c|}{ Presente } & \multicolumn{2}{|c|}{ Ausente } & \\
\hline & $\mathbf{n}$ & Média $( \pm D P)$ & $\mathbf{n}$ & Média ( \pm DP) & \\
\hline Idade & 69 & $50(15,48)$ & 11 & $31,55(10,34)$ & $p=0,000^{1}$ \\
\hline Anos de estudo & 69 & $6,62(4,85)$ & 11 & $7,55(5,12)$ & $p=0,563^{1}$ \\
\hline Renda familiar & 69 & 2,37 & 11 & 1,90 & $p=0,534^{2}$ \\
\hline Dor & 69 & 2,04 & 11 & 3,36 & $\mathrm{p}=0,170^{2}$ \\
\hline
\end{tabular}

Legenda: ${ }^{2}$ Teste T assumindo variâncias iguais; ${ }^{2}$ Teste de Mann-Whitney

Conforme observado, o risco de quedas foi identificado com maior frequência em pessoas do sexo masculino $(48,75 \%)$, com uma média de $50( \pm 15,48)$ anos de idade e $6,62( \pm 4,85)$ anos de estudo. Ao comparar as características da amostra, de acordo com o verificado, os indivíduos com o DE em questão apresentavam maior média de idade e renda familiar, bem como um menor tempo de estudo. A maior parte desses pacientes alegou viver com um companheiro $(63,75 \%)$ e ter uma religião a qual praticava (68,75\%). Quanto à intensidade da dor referida, os pacientes que não foram diagnosticados com o risco de quedas tiveram maior média de dor.

Concernente ao cruzamento entre o diagnóstico Risco de quedas e os dados sociodemográficos descritos foi verificada associação significativa com a religião (associação fraca, $\mathrm{o}=-0,339$, $\mathrm{p}=0,005)$. Neste caso, os pacientes praticantes de alguma religião têm $86 \%$ menos chance de apresentar o Risco de quedas, embora, sob o ponto de vista clínico, não se perceba uma relação entre estas variáveis. De acordo com o apurado, merece destaque a associação significante entre a idade e a ocorrência do diagnóstico Risco de quedas $(p=0,000)$. 
A seguir, estão descritos os fatores de risco mais prevalentes segundo as categorias investigadas (exclusivos em adultos, fisiológicos, e medicamentosos), bem como sua relação associativa com o DE Risco de quedas. Na Tabela 4 são relacionados os fatores de risco exclusivos de adultos.

Conforme observado, viu-se que os fatores de risco História de quedas (20\%) e Idade acima de 65 anos (15\%) foram os mais expressivos em sua categoria. Não foi identificada associação estatisticamente significativa entre os fatores de risco específicos para adultos.

A tabela 4 apresenta os fatores de risco fisiológicos. Foram identificados 12 fatores de risco nessa categoria, sendo Condições pósoperatórias (83,8\%) e Anemia (33,8\%) os que mais se destacaram. Notou-se, ainda, associação estatística significativa entre o fator de risco Condições pós-operatórias e o DE Risco de quedas (associação moderada, $\mathrm{o}=0,513, \mathrm{p}=$ 0,000). Tal associação sugere que pacientes em período pós-operatório apresentam 18 vezes mais chance de desenvolver o risco de quedas. Se nesta pesquisa fosse considerado um nível de significância de $10 \%$, a condição de anemia e o DE Risco de quedas apresentariam associação estatisticamente significante.

Tabela 3 - Distribuição dos pacientes em pós-operatório, de acordo com os fatores de risco específicos para adultos e a ocorrência do diagnóstico de enfermagem Risco de quedas. Natal-RN, 2013

\begin{tabular}{|c|c|c|c|c|}
\hline Fatores de Risco & $\mathbf{N}$ & $\%$ & $\mathrm{IC}^{1}(95 \%)$ & $\begin{array}{c}\text { Estatísticas associativas com o DE } \\
\text { Risco de quedas }\end{array}$ \\
\hline $\begin{array}{l}\text { História de } \\
\text { quedas }\end{array}$ & 16 & 20 & $11,9-30,4$ & $\begin{array}{c}p=0,302^{2} \\
\text { Phi }=0,109(p=0,330) \\
O R=2,777(0,328-23,463)\end{array}$ \\
\hline $\begin{array}{l}\text { Idade acima de } \\
65 \text { anos }\end{array}$ & 12 & 15 & $8,0-24,7$ & $\begin{array}{c}p=0,146^{2} \\
\text { Phi }=0,168(p=0,134) \\
O^{*}\end{array}$ \\
\hline $\begin{array}{l}\text { Uso de cadeira } \\
\text { de rodas }\end{array}$ & 5 & 6,3 & $2,1-14$ & $\begin{array}{c}p=0,467^{2} \\
\text { Phi }=0,103(p=0,356) \\
\text { OR }^{*}\end{array}$ \\
\hline $\begin{array}{l}\text { Uso de } \\
\text { dispositivos } \\
\text { auxiliares }\end{array}$ & 2 & 2,5 & $0,3-8,7$ & $\begin{array}{c}p=0,742^{2} \\
\text { Phi }=0,064(p=0,567) \\
O^{*}\end{array}$ \\
\hline
\end{tabular}

DE- Diagnóstico de enfermagem; Phi - Coeficiente Fi; OR - Razão de chance; OR*- Razão de chance não definida; 1 IC - Intervalo de Confiança; ${ }^{2}$ - Teste exato de Fisher.

Tabela 4 - Distribuição dos pacientes em pós-operatório, segundo os fatores de risco fisiológicos e a ocorrência do diagnóstico de enfermagem Risco de quedas. Natal-RN, 2013

\begin{tabular}{|c|c|c|c|c|}
\hline Fatores de Risco & $\mathbf{N}$ & $\%$ & $I^{1}(95 \%)$ & $\begin{array}{c}\text { Estatísticas associativas com o DE } \\
\text { Risco de quedas }\end{array}$ \\
\hline $\begin{array}{l}\text { Condições } \\
\text { pós-operatórias }\end{array}$ & 67 & 83,8 & $73,8-91,1$ & $\begin{array}{c}p=0,000^{2} \\
\text { Phi }=0,513(p=0,000) \\
O R=18,375(4,154-81,267)\end{array}$ \\
\hline Anemia & 27 & 33,8 & $23,6-45,2$ & $\begin{array}{c}p=0,057^{2} \\
\text { Phi }=0,208(p=0,063) \\
O R=6,046(0,731-50,000)\end{array}$ \\
\hline $\begin{array}{l}\text { Mobilidade física } \\
\text { prejudicada }\end{array}$ & 20 & 25 & $16,0-35,9$ & $\begin{array}{c}p=0,555^{2} \\
\text { Phi }=-0,021(p=0,851) \\
O R=0,871(0,207-3,663)\end{array}$ \\
\hline $\begin{array}{l}\text { Equilíbrio } \\
\text { prejudicado }\end{array}$ & 16 & 20 & $11,9-30,4$ & $\begin{array}{c}p=0,070^{2} \\
\text { Phi }=0,200(p=0,074) \\
O^{*}\end{array}$ \\
\hline $\begin{array}{l}\text { Vertigem ao virar } \\
\text { o pescoço }\end{array}$ & 15 & 18,8 & $10,9-29$ & $\begin{array}{c}p=0,085^{2} \\
\text { Phi }=0,192(p=0,086) \\
O^{*}\end{array}$ \\
\hline
\end{tabular}




\begin{tabular}{|c|c|c|c|c|}
\hline $\begin{array}{l}\text { Dificuldades } \\
\text { visuais }\end{array}$ & 11 & 13,8 & $7,1-23,3$ & $\begin{array}{c}p=0,174^{2} \\
\mathrm{Phi}=0,159(p=0,154) \\
\text { OR }^{*}\end{array}$ \\
\hline Neoplasias & 5 & 6,3 & $2,1-14$ & $\begin{array}{c}\mathrm{p}=0,467^{2} \\
\mathrm{Phi}=0,103(\mathrm{p}=0,356) \\
\text { OR }^{*}\end{array}$ \\
\hline Falta de sono & 4 & 5 & $1,4-12,3$ & $\begin{array}{c}p=0,546^{2} \\
\text { Phi }=0,092(p=0,413) \\
\text { OR }^{*}\end{array}$ \\
\hline $\begin{array}{l}\text { Força diminuída } \\
\text { nas extremidades } \\
\text { inferiores }\end{array}$ & 4 & 5 & $1,4-12,3$ & $\begin{array}{c}p=0,546^{2} \\
\text { Phi }=0,092(p=0,413) \\
\text { OR }^{*}\end{array}$ \\
\hline Doença vascular & 4 & 5 & $1,4-12,3$ & $\begin{array}{c}p=0,546^{2} \\
\mathrm{Phi}=0,092(p=0,413) \\
\text { OR }^{*}\end{array}$ \\
\hline $\begin{array}{l}\text { Problemas nos } \\
\text { pés }\end{array}$ & 3 & 3,8 & $0,8-10,6$ & $\begin{array}{c}\mathrm{p}=0,637^{2} \\
\text { Phi }=0,079(p=0,481) \\
\text { OR* }^{*}\end{array}$ \\
\hline $\begin{array}{l}\text { Dificuldades } \\
\text { auditivas }\end{array}$ & 1 & 1,3 & $0-6,8$ & $\begin{array}{c}p=0,862^{2} \\
\text { Phi }=0,045(p=0,688) \\
\text { OR }^{*}\end{array}$ \\
\hline
\end{tabular}

DE- Diagnóstico de enfermagem; Phi - Coeficiente Fi; OR - Razão de chance; OR*- Razão de chance não definida; 1 IC - Intervalo de Confiança; ${ }^{2}$ Teste exato de Fisher.

Uma vez encontrada relação entre a condição pós-operatória e o risco de quedas, foi realizado o cruzamento entre o tipo de cirurgia e o DE em questão, conforme descrito na tabela 5. No entanto, não foi encontrada associação estatística significante, demonstrando que a relação encontrada é referente ao estado geral do paciente no pósoperatório e não quanto ao tipo de cirurgia em si.

Com relação aos fatores de risco medicamentosos exibidos na tabela 6 , a maioria dos pacientes (55\%) estava em uso de narcóticos e/ou opiáceos, seguido do uso de agentes antihipertensivos (43,8\%) e inibidores da ECA (21,3\%). Não foi identificada associação estatisticamente significante entre o uso de narcóticos/opiáceos e o DE Risco de quedas. Por outro lado, contatouse associação estatística significativa entre o uso de agentes anti-hipertensivos (associação fraca, o $=0,279, p=0,011)$. Segundo a razão de chance, esta associação indica que os pacientes em uso de anti-hipertensivos apresentam 9 vezes mais chance de desenvolver o risco de quedas.

Tabela 5 - Ocorrência do Diagnóstico de Enfermagem Risco de quedas. Natal-RN, 2013

\begin{tabular}{lccc}
\hline Tipo de cirurgia & $\mathbf{N}$ & $\mathbf{\%}$ & $\begin{array}{l}\text { Estatística associativa com } \\
\text { o DE Risco de quedas }\end{array}$ \\
\hline Digestiva/urológica & 56 & 70 & $\mathrm{p}=0,430^{1}$ \\
\hline Cabeça e pescoço & 10 & 12,50 & $\mathrm{p}=0,135^{1}$ \\
\hline Torácica & 8 & 10 & $\mathrm{p}=0,6971$ \\
\hline Ortopédica & 5 & 6,25 & $\mathrm{p}=0,4671$ \\
\hline Vascular & 1 & 1,25 & $\mathrm{p}=0,862^{1}$ \\
\hline
\end{tabular}

1- Teste exato de Fisher. 
Tabela 6 - Distribuição dos pacientes em pós-operatório de acordo com os fatores de risco medicamentosos e a ocorrência do diagnóstico de enfermagem Risco de quedas. Natal-RN, 2013

\begin{tabular}{|c|c|c|c|c|}
\hline Fatores de Risco & $\mathbf{N}$ & $\%$ & $\mathrm{IC}^{1}(95 \%)$ & $\begin{array}{l}\text { Estatísticas associativas com o DE } \\
\text { Risco de quedas }\end{array}$ \\
\hline $\begin{array}{l}\text { Narcóticos/ } \\
\text { opiáceos }\end{array}$ & 44 & 55 & $43,5-66,2$ & $\begin{array}{c}\mathrm{p}=0,156^{2} \\
\mathrm{Phi}=0,150(\mathrm{p}=0,181) \\
\mathrm{OR}=2,413(0,646-9,019)\end{array}$ \\
\hline $\begin{array}{l}\text { Agentes anti- } \\
\text { hipertensivos }\end{array}$ & 35 & 43,8 & $32,7-55,3$ & $\begin{array}{c}p=0,011^{2} \\
\text { Phi }=0,279(p=0,013) \\
O R=9,714(1,178-80,057)\end{array}$ \\
\hline $\begin{array}{l}\text { Inibidores da } \\
\text { ECA (enzima } \\
\text { conversora da } \\
\text { angiotensina) }\end{array}$ & 17 & 21,3 & $12,9-31,8$ & $\begin{array}{c}p=0,058^{2} \\
\mathrm{Phi}=0,207(p=0,064) \\
\mathrm{OR}^{*}\end{array}$ \\
\hline Diuréticos & 12 & 15 & $8,0-24,7$ & $\begin{array}{c}p=0,479^{2} \\
\text { Phi }=0,066(p=0,555) \\
\text { OR=1,896 }(0,220-16,352)\end{array}$ \\
\hline $\begin{array}{l}\text { Agentes } \\
\text { ansiolíticos }\end{array}$ & 3 & 3,8 & $0,8-10,6$ & $\begin{array}{c}\mathrm{p}=0,637^{2} \\
\mathrm{Phi}=0,079(\mathrm{p}=0,481) \\
\mathrm{OR}^{*}\end{array}$ \\
\hline
\end{tabular}

DE- Diagnóstico de enfermagem; Phi - Coeficiente Fi; OR - Razão de chance; OR*- Razão de chance não definida; 1 IC - Intervalo de Confiança; ${ }^{2}$ Teste exato de Fisher.

\section{DISCUSSÃO}

Com referência aos achados desta investigação, a literatura mostra que, de forma geral, o indivíduo quando hospitalizado apresenta um desequilíbrio peculiar de suas necessidades humanas básicas, e esta condição é agravada pela condição pós-operatória ${ }^{(12)}$. Os pacientes em período pós-operatório encontram-se muitas vezes em uso de diversos dispositivos hospitalares, como suporte de soro, acesso venoso, sonda vesical, bolsa coletora de urina e drenos, além da presença da própria incisão cirúrgica. Estes fatores podem dificultar a deambulação, bem como a realização do autocuidado e ocasionar severos desdobramentos à recuperação do indivíduo, como as quedas, destacadas nesta pesquisa.

Entre os pacientes incluídos no estudo $86,25 \%$ apresentaram o DE Risco de quedas, em sua maioria homens. Esse dado contradiz os resultados de um estudo de coorte retrospectivo realizado no Texas ${ }^{(13)}$, no qual a proporção de mulheres foi maior. Por outro lado, um estudo prospectivo observacional que acompanhou os fatores de risco para quedas desde o pré até o pós-operatório apontou para uma maior quantidade de homens no estudo ${ }^{(14)}$. Percebe-se então o foco das pesquisas em buscar a relação entre características clínicas e comportamentais da população ao invés de relacionar o risco de quedas com o gênero ${ }^{(7)}$.

Com referência a associação encontrada entre a prática religiosa e uma menor chance de apresentar o risco de queda, não foi encontrado na literatura, sob o ponto de vista clínico, uma razão que justificasse tal associação.

Concernente à relação entre idade e o DE Risco de quedas ora identificada, relação semelhante foi encontrada em um estudo realizado no Reino Unido, no qual foi observado que indivíduos em uma maior faixa etária ficam mais expostos ao risco de quedas ${ }^{(15)}$. Pesquisas mostram que ao envelhecer as pessoas apresentam maior tendência à instabilidade postural, alterações de marcha, bem como enrijecimento das articulações musculoesqueléticas ${ }^{(16-17)}$. As alterações sofridas pelo avanço da idade vinculada à condição pósoperatória favorecem ao possível evento queda.

Sobre o Risco de quedas e os fatores de risco específicos para adultos, obteve destaque o fator História de quedas. Um estudo executado para comparar demográfica, clínica e funcionalmente idosos institucionalizados com e sem episódios de quedas, mostrou que a ocorrência de uma ou mais quedas no ano anterior resulta em aumento de reincidência deste evento ${ }^{(18)}$.

Relativo à associação estatística significativa encontrada entre a condição pós-operatória e o DE Risco de quedas, conforme revelado por um 
estudo de coorte realizado pela Universidade do Colorado ${ }^{(5)}$, as quedas do cliente em pósoperatório tiveram como causa a fraqueza, a perda do equilíbrio, até mesmo a síncope. Este fato denota uma maior relação entre a condição pós-operatória e a vulnerabilidade aumentada do indivíduo ao risco de quedas em detrimento ao tipo de cirurgia realizada.

Outra característica apontada neste mesmo estudo foi a presença da anemia como fator predisponente a queda. Quanto a isto, sabe-se que a anemia pode ser definida como a diminuição da concentração de hemoglobina do sangue. Esta condição pode representar um desequilíbrio da associação de oxigênio com este componente e apontar consequências clínicas como dispneia, fraqueza, letargia, baixa perfusão tissular e até mesmo confusão mental, consideradas possíveis propensores de uma queda ${ }^{(19)}$.

Os resultados apontaram que mais de $50 \%$ da amostra estava em uso de medicações da ordem de narcóticos e/ou opiáceos. Um estudo de caso-controle ${ }^{(20)}$ revelou associação entre o uso de analgésicos narcóticos e o risco de quedas em pacientes com osteoartrite. Outra classe medicamentosa de destaque foi a classe dos antihipertensivos. Em uma pesquisa cujo objetivo foi verificar a relação entre o uso de medicamentos e o risco para fratura por queda no idoso, foi identificado que os agentes hipotensores podem provocar hipotensão postural, sonolência, vertigem, polaciúria, dentre outros efeitos, os quais podem propiciar o acontecimento de quedas ${ }^{(21)}$.

Ao identificar e comparar com outras pesquisas sobre fatores de risco do DE Risco de quedas em pacientes no pós-operatório, reúne-se subsídios norteadores para a elaboração de ações que previnam esses episódios indesejados, por exemplo, projetos de orientação pré-operatória, proporcionando diminuição da ansiedade, maior conforto e segurança para os pacientes em internamento cirúrgico, contribuindo para a diminuição de agravos e conservação da qualidade do atendimento hospitalar ${ }^{(4)}$.

\section{CONSIDERAÇÕES FINAIS}

Em síntese, mediante a caracterização do DE Risco de quedas em pacientes no período pósoperatório, percebeu-se que os fatores de risco que mais se destacaram foram: história de quedas, condições pós-operatórias, uso de narcóticos e/ou opiáceos e uso de anti-hipertensivos. Dentre estes fatores, ressaltam-se as associações estatísticas entre a condição pós-operatória e o DE Risco de quedas (associação moderada), bem como entre o uso de antihipertensivos e o referido diagnóstico (associação fraca).

Diante da diversidade de fatores de risco encontrados em pacientes no período pósoperatório torna-se imperativo a identificação precoce desses por profissionais de saúde, a fim de propor ações preventivas e eficazes que suprimam ou minimizem tais riscos.

O presente estudo apresentou algumas limitações pelo próprio delineamento adotado. Trata-se de um desenho transversal, o qual possibilita a identificação de associações, mas não consegue estabelecer relação causal. Portanto, por se tratar de um fenômeno de risco, sugere-se a execução de posteriores pesquisas de desenho longitudinal, quer seja prospectiva ou retrospectiva, no sentido de estabelecer uma relação de causalidade entre estes fatores de risco e o diagnóstico, bem como saber quais são os fatores mais preponderantes em provocar o evento risco de quedas. Aconselhase ainda, outras pesquisas em cenários diversos, principalmente com o objetivo de comparação com os resultados encontrados neste estudo.

Outra limitação encontrada foi referente à identificação dos fatores ambientais, percebeu-se uma divergência entre as interpretações por parte dos pesquisadores quanto a essa questão. Como esse quesito não foi padronizado no questionário decidiu-se excluir essa categoria do resultado final. Recomendamos, então, a inclusão desta categoria em estudos futuros.

Com base nestes achados, recomenda-se também o desenvolvimento e validação de instrumentos acurados e precisos para avaliação da situação de saúde do paciente com relação ao risco de quedas, além de quantificar a presença dos fatores de risco no estado de saúde do indivíduo. Desta forma, a enfermagem poderá atuar de forma direcionada e enfocar suas ações para prevenção do evento queda no período pós-operatório.

\section{REFERÊNCIAS}

1. Vargas MAO, Luz AMH. Práticas seguras do/no cuidado de enfermagem no contexto hospitalar: é preciso pensar sobre isso e aquilo. Enferm. Foco. 2010; 1(1):23-7. 
2. Smeltzer SC, Bare BG, Hinkle JL, Cheever KH. Tratado de Enfermagem Médico-Cirúrgico. $12^{\mathrm{a}}$ ed. Rio de Janeiro: Guanabara Koogan; 2012.

3. Davenport RD, Vaidean GD, Jones CD, Chandler AM, Kessler LA, Mion LC, et al. Falls following discharge after an in-hospital fall. BMC Geriatrics. 2009; 9(53):1-17.

4. Clarke HD, Timm VL, Goldberg BR, Hattrup SJ. Preoperative patient education reduces in-hospital falls after total knee arthroplasty. Clin Orthop Relat Res. 2012; 470(1):244-9.

5. Church S, Robinson TN, Angles EM, Tran ZV, Wallace JI. Postoperative falls in the acute hospital setting: characteristics, risk factors, and outcomes in males. Am J Surg. 2011; 201(2): 197-202.

6. Cooper CL, Nolt JD. Development of an evidencebased pediatric fall prevention program. J Nurs Care Quat. 2007; 22(2):107-12.

7. Vitor AF, Lopes MVO, Araujo TL. Diagnóstico de enfermagem Risco de Quedas em pacientes com angina instável. Rev. Rene. 2010; 11(1):105-13.

8. Garcia TL, Nóbrega MML. Processo de enfermagem: da teoria à prática assistencial e de pesquisa. Esc Anna Nery Rev Enferm. 2009; 13(1): 188-93.

9. North American Nursing Diagnosis Association. Diagnósticos de enfermagem da NANDA: definições e classificação 2012-2014. Porto Alegre: Artmed; 2013.

10. Gordon M. Nursing diagnosis: process and application. St. Louis: Mosby; 1994.

11. Ministério da Saúde (BR). Conselho Nacional de Saúde. Resolução 196, de 10 de outubro de 1996. Dispõe sobre diretrizes e normas regulamentadoras de pesquisas envolvendo seres humanos. Brasília; 1996.

12. Jorgetto GV, Noronha R, Araújo IEM. Assistência de enfermagem a pacientes cirúrgicos: avaliação comparativa. Rev. Eletr. Enf. [Internet] 2005; 7(3) [acesso em 10 abr 2013]. Disponível: http://www.fen. ufg.br/revista/revista7_3/pdf/original_03.pdf

13. Chatterjee S, Chen H, Johnson ML, Aparasu RR. Risck of falls and fractures in older adults using atypical antipsychotic agents: a propensity score-adjusted, retrospective cohort study. Am J Geriatr Pharmacother. 2012; 10(2): 83- 94.

14. Swinkels A, Newman JH, Allain TJ. A prospective observational study of falling before and after knee replacement surgery. Age Ageing. 2009; 38(2):175-81.

15. Armstrong ME, Cairns BJ, Banks E, Green J, Reeves GK, Beral V. Different effects of age, adiposity and physical activity on the risk of ankle, wrist and rip fractures in postmenopausal women. Bone. 2012; 50(6): 1394-400.
16. Vasconcelos FF, Araújo TL, Moreira TMM, Lopes MVO. Association among nursing diagnoses, demographic variables, and clinical characteristics of patients with high blood pressure. Acta Paul. Enferm. 2007; 20(3): 326-32.

17. Grden CRB, Sousa JAV, Lenardt MH, Pesck RM, Seima MD, Borges PKO. Caracterização de idosos vítimas de acidentes por causas externas. Cogitare enferm. [Internet] 2014; 19(3) [acesso em 04 nov 2014]. Disponível: http://ojs.c3sl.ufpr.br/ojs/index.php/ cogitare/article/viewFile/37972/23225

18. Teixeira DC, Oliveira IL, Dias RC. Perfil demográfico, clínico e funcional de idosos institucionalizados com história de quedas. Fisioter. Mov. 2006; 19(2):101-08.

19. Hoffbrand AV, Moss PAH. Fundamentos em hematologia. $6^{a}$ ed. Porto Alegre: Artmed; 2013.

20. Rolita L, Spegman A, Tang X, Cronstein BN. Greater Number of Narcotic Analgesic Prescriptions for Osteoarthritis Is Associated with Falls and Fractures in Elderly Adults. J. Am. Geriatr. Soc. 2013; 61(3):335-40.

21. Hamra A, Ribeiro MB, Miguel OM. Correlação entre fratura por queda em idosos e uso prévio de medicamentos. Acta Ortop. Bras. 2007; 15(3):143-5. 